\title{
Effect of hyperbaric oxygenation on mitochondrial function of neuronal cells in the cortex of neonatal rats after hypoxic-ischemic brain damage
}

\author{
L. Yang, M.Y. Hei, J.J. Dai, N. Hu and X.Y. Xiang \\ Department of Pediatrics, Third Xiangya Hospital, Central South University, Changsha, Hunan, China
}

\begin{abstract}
The timing and mechanisms of protection by hyperbaric oxygenation (HBO) in hypoxic-ischemic brain damage (HIBD) have only been partially elucidated. We monitored the effect of $\mathrm{HBO}$ on the mitochondrial function of neuronal cells in the cerebral cortex of neonatal rats after HIBD. Neonatal Sprague-Dawley rats (total of 360 of both genders) were randomly divided into normal control, HIBD, and HIBD + HBO groups. The HBO treatment began immediately after hypoxia-ischemia (HI) and continued once a day for 7 consecutive days. Animals were euthanized 0, 2, 4, 6, and $12 \mathrm{~h}$ post-HI to monitor the changes in mitochondrial membrane potential $(\Delta \Psi \mathrm{m})$ occurring soon after a single dose of HBO treatment, as well as $2,3,4,5,6$, and 7 days post- $\mathrm{HI}$ to study $\Delta \Psi \mathrm{m}$ changes after a series of $\mathrm{HBO}$ treatments. Fluctuations in $\Delta \Psi \mathrm{m}$ were observed in the ipsilateral cortex in both HIBD and HIBD + HBO groups. Within 2 to $12 \mathrm{~h}$ after $\mathrm{HI}$ insult, the $\triangle \Psi \mathrm{m}$ of the HIBD and HIBD $+\mathrm{HBO}$ groups recovered to some extent. A secondary drop in $\triangle \Psi \mathrm{m}$ was observed in both groups during the 1-4 days post-HI period, but was more severe in the HIBD + HBO group. There was a secondary recovery of $\Delta \Psi \mathrm{m}$ observed in the HIBD $+\mathrm{HBO}$ group, but not in the HIBD group, during the 5-7 days period after $\mathrm{HI}$ insult. HBO therapy may not lead to improvement of neural cell mitochondrial function in the cerebral cortex in the early stage post-HI, but may improve it in the sub-acute stage post-HI.
\end{abstract}

Key words: Hyperbaric oxygenation; Hypoxia-ischemia; Brain; Mitochondrial function; Neonatal; Rat

\section{Introduction}

Medical research has long focused on understanding the pathophysiology of hypoxic-ischemic brain damage (HIBD) in newborns, which still has a high incidence in the world, causing negative developmental outcomes in children (1). Clinically, though hypothermia has been proved to be effective in reducing hypoxia-ischemia $(\mathrm{HI})$ induced brain damage $(2,3)$, specific treatment to HIBD is still limited, leaving the task of finding new therapeutic methods to be very necessary. The initial insult in HIBD is the deprivation of oxygen $\left(\mathrm{O}_{2}\right)$ to the brain cells. There is a cascade of brain cell damage after $\mathrm{HI}$ insult, from $\mathrm{O}_{2}$ deprivation, followed by $\mathrm{N}$-methyl-D-aspartate (NMDA) receptor activation and intracellular free calcium accumulation, to mitochondrial dysfunction and other biochemical changes of brain cells, finally resulting in the death of cells via apoptosis or necrosis (4). Any intervention that effectively reverses each step in the cascade may have a potential treatment effect in HIBD. Interventions directed to improving mitochondrial function of injured brain cells has drawn special attention (5). Under any circumstance of hypoxia/ischemia-inducing cell death, mitochondrial dysfunction caused by energy failure is the earliest pathophysiology process $(6,7)$. The usual pathway is: energy failure - free calcium accumulation in the cell - mitochondrial dysfunction - cytochrome $\mathrm{C}$ released from the mitochondria - other cascade steps of cell damage - cell necrosis/apoptosis.

Theoretically, hyperbaric oxygenation (HBO) could affect the recovery of mitochondrial function in HIBD, since such a hyperoxic condition will undoubtedly affect cerebral energy metabolism, in which mitochondria plays critical roles mediating cellular responses to $\mathrm{HI}$ stress. In the past, $\mathrm{HBO}$ has been shown to increase cerebral blood flow and decrease intracranial pressure while increasing oxygen availability to injured brain cells (8). An animal study found that $\mathrm{HBO}$ appears to restore mitochondrial function by greatly increasing $\mathrm{O}_{2}$ delivery diffusion gradient, which subsequently improves cerebral aerobic metabolism after brain injury (9). We hypothesize that $\mathrm{HBO}$ may reduce $\mathrm{HI}$-induced brain injury by affecting the brain cell mitochondrial function. Our aim is also to evaluate the change patterns of mitochondrial function in

Correspondence: M.Y. Hei: <1797976511@qq.com> 
HBO treatment. In the present study, we used flow cytometer to explore the change of mitochondrial membrane potential $(\Delta \Psi \mathrm{m})$ of neuronal cells in the cortex of neonatal rats after HIBD.

\section{Material and Methods}

The present study was approved by the Medical Ethics Committee of the Third Xiangya Hospital. The study was carried out according to the guidelines for animal experimentation at Central South University, Changsha, Hunan, China.

\section{Animals and HIBD model}

Sprague Dawley rat pups on postnatal day 7 (total of 360 , of both genders, mean weight: $12.11 \pm 1.19 \mathrm{~g}$ ) were randomly divided into three groups: normal control group, HIBD group, and HIBD + HBO group. The Rice-Vannucci model of HIBD (10) was established with minor modifications. Briefly, the left common carotid artery was permanently ligated under isoflurane inhalation anesthesia (10). After resting with its dam for $2 \mathrm{~h}$ at room temperature, the pup was subjected to humidified $8 \% \mathrm{O}_{2} / 92 \% \mathrm{~N}_{2}$ hypoxia in an air-tight chamber at $34^{\circ} \mathrm{C}$ for $2 \mathrm{~h}$ (10 pups at a time). The end of $\mathrm{HI}$ was considered to be $0 \mathrm{~h}$ time-point. This technique has been successfully used in our laboratory for years in a series of HIBD studies (11-13).

\section{HBO treatment}

Series of $\mathrm{HBO}$ treatment began after the end of $\mathrm{HI}$ insult. Animals (10 pups at a time) were placed in a pressure chamber made of transparent acrylic plastic (inner diameter $25 \mathrm{~cm}$, length $50 \mathrm{~cm}$ ). The temperature in the chamber was kept at $34^{\circ} \mathrm{C}$. HBO-treated animals were pressurized for $15 \mathrm{~min}$ to 2-atmosphere absolute pressure (ATA) $\left(0.2 \mathrm{MPa}\right.$ ) and maintained for $60 \mathrm{~min}$ at $\mathrm{O}_{2}$ concentration of no less than $85 \%$. Controls were also transferred into the chamber for the same duration, but not pressurized, and with normal room air. The HBO treatment was given once a day for 7 consecutive days (in 24-h intervals).

\section{Flow cytometer measurement of $\Delta \Psi \mathrm{m}$}

Animals were euthanized by decapitation after giving overdose of phenobarbital, at $0,2,4,6$ and $12 \mathrm{~h}$, in order to study the $\triangle \Psi \mathrm{m}$ changes at the very early stage after a single dose of $\mathrm{HBO}$ treatment, and at 2, 3, 4, 5, 6 and 7 days, in order to study the $\Delta \Psi \mathrm{m}$ changes after a series of $\mathrm{HBO}$ treatments. Cell suspension of the cerebral cortex was prepared as described previously (14). In detail, cerebral cortex was separated and immersed in Earl's balanced salt iced solution, and then transferred to $2 \mathrm{~mL}$ of papain digesting solution. The tissues were minced, put on a shaker at $37^{\circ} \mathrm{C}$ and gently shaken. After $30 \mathrm{~min}$, an equal volume of normal physiological medium/bovine serum albumin (NPM/ BSA) was added to block the reaction. The mixture was gently triturated with $5 \mathrm{~mL}$ pipette over 10 passages. The cell suspension was kept upright on ice for a while and any undigested or aggregated particles were removed after. The solution was centrifuged at a low speed of $300 \times g$ at $4^{\circ} \mathrm{C}$ in an Eppendorf centrifuge 5810R (Germany) for $5 \mathrm{~min}$. The supernatant was decanted and the cells were re-suspended in $5 \mathrm{~mL}$ of NPM/BSA. After 3 rounds of centrifugation/ re-suspension, dissociated cells were finally re-suspended at a density of $2 \times 10^{6}$ cells $/ \mathrm{mL}$ in NPM/BSA. The counting for viable cells was done after trypan-blue exclusion.

The $\Delta \Psi \mathrm{m}$ was determined by measuring the green fluorescence of Rhodamine123 (Rho123; Sigma, USA). Mitochondria were stained by directly adding a stock solution of Rho123 (10 $\mu \mathrm{M}$ in ethanol) to $1 \mathrm{~mL}$ of the cell suspension to a final concentration of $1 \mu \mathrm{M}$ for $45 \mathrm{~min}$ with gentle horizontal agitation in a shaker at $37^{\circ} \mathrm{C}$ under an atmosphere of $95 \% \mathrm{O}_{2} / 5 \% \mathrm{CO}_{2}$. Changes in Rho123 fluorescence were measured by flow cytometer (excitation wavelength at $488 \mathrm{~nm}$ and emission wavelength at 525 nm; Cytomics FC500, Beckman Coulter, Inc., USA). As a positive control for $\Delta \Psi \mathrm{m}$ depolarization, $1 \mathrm{~mL}$ of cell suspension from a randomly chosen animal was preincubated with the uncoupling agent cyanide m-chlorophenylhydrazone (mCICCP, $100 \mu \mathrm{M}$, Sigma, USA) before loading Rho123.

\section{Statistical analysis}

Data were analyzed using the SPSS 13.0 software (USA). ANOVA was used to evaluate differences among experimental groups followed by Tukey post hoc test. Statistical significance was set at $\mathrm{P}<0.05$.

\section{Results}

\section{Fluctuating changes of $\Delta \Psi \mathrm{m}$ after a single dose of HBO within $24 \mathrm{~h}$}

The mean $\Delta \Psi \mathrm{m}$ of the normal control group at matched time-points within $24 \mathrm{~h}$ was $(4.72 \pm 0.12) \mathrm{MFL}$ (mean fluorescence level). In the HIBD and HIBD + HBO groups, the $\Delta \Psi \mathrm{m}$ of the ipsilateral cortex at each timepoint within $24 \mathrm{~h}$ was lower than the normal control, and the lowest one was at $0 \mathrm{~h}$ in both groups. There was a significant difference between the HIBD and normal control groups, and between the HIBD + HBO and normal control groups. The $\Delta \Psi \mathrm{m}$ in both groups had a fluctuating change pattern from 0 to $12 \mathrm{~h}$, showing first a decrease, then a recovery at $2 \mathrm{~h}$, and a decrease again at $12 \mathrm{~h}$. The mean $\triangle \Psi \mathrm{m}$ in the HIBD $+\mathrm{HBO}$ group was higher than that in the HIBD group at 2, 4, and $6 \mathrm{~h}$, but lower than that in the HIBD group at 0,12 and $24 \mathrm{~h}$ (Table 1). Among these differences, significance was found at 4,12 , and $24 \mathrm{~h}$.

\section{Recovering $\Delta \Psi \mathrm{m}$ changes after daily $\mathrm{HBO}$ treatment for 7 days}

The mean $\Delta \Psi \mathrm{m}$ for the normal control group at matched time-points from 1-7 days after $\mathrm{HI}$ was $4.66 \pm 0.80 \mathrm{MFL}$, 
Table 1. Mitochondrial membrane potential (in mean fluorescence level, MFL) in ipsilateral cortex after a single dose of hyperbaric oxygenation (HBO) during $24 \mathrm{~h}$ after hypoxic-ischemic brain damage (HIBD).

\begin{tabular}{lcccrcc}
\hline Time-point & Normal control & HIBD group & HIBD + HBO group & $t$ value & $\mathrm{P}$ value & $95 \% \mathrm{Cl}$ \\
\hline $0 \mathrm{~h}$ & $4.72 \pm 0.12$ & $2.85 \pm 0.56$ & $2.58 \pm 1.06$ & 0.71 & 0.488 & $-0.53 ; 1.07$ \\
$2 \mathrm{~h}$ & $4.66 \pm 0.80$ & $3.92 \pm 2.58$ & $4.03 \pm 1.77$ & -0.11 & 0.918 & $-2.28 ; 2.07$ \\
$4 \mathrm{~h}$ & $4.78 \pm 0.13$ & $4.21 \pm 0.37$ & $4.88 \pm 0.37$ & -4.04 & 0.001 & $-1.02 ;-0.32$ \\
$6 \mathrm{~h}$ & $4.77 \pm 0.21$ & $4.08 \pm 0.98$ & $4.68 \pm 1.55$ & -1.04 & 0.311 & $-1.82 ; 0.61$ \\
$12 \mathrm{~h}$ & $4.61 \pm 0.45$ & $4.15 \pm 0.15$ & $3.87 \pm 0.06$ & 4.92 & 0.001 & $0.16 ; 0.42$ \\
$24 \mathrm{~h}$ & $4.69 \pm 0.56$ & $3.73 \pm 0.36$ & $3.09 \pm 0.48$ & 3.25 & 0.005 & $0.22 ; 1.05$ \\
\hline
\end{tabular}

Data are reported as means $\pm S D$ ( $n=10$ in each group at each time-point) (ANOVA followed by Tukey's post hoc test).

which was not significantly different from that within $24 \mathrm{~h}$ after $\mathrm{HI}$. At 1 to 7 days, the $\Delta \Psi \mathrm{m}$ of the ipsilateral cortex of the HIBD group was significantly lower than the normal control group without much difference between each other. The $\Delta \Psi \mathrm{m}$ of the HIBD $+\mathrm{HBO}$ group at 1,2 , and 3 days was initially lower than of the HIBD group, then it recovered at 4 and 5 days, and it became higher at 6 and 7 days. The difference between the HIBD group and the HIBD $+\mathrm{HBO}$ group was significant at $1,2,3,6$, and 7 days $(P<0.05)$ (Table 2). The $\Delta \Psi \mathrm{m}$ of the HIBD $+\mathrm{HBO}$ group at 6 and 7 days was very similar to the normal control $(P>0.05)$.

The change of $\Delta \Psi \mathrm{m}$ in the ipsilateral cortex from the normal control, HIBD and HIBD + HBO groups at each time-point is summarized in Figure 1. There was a "droprecovery-secondary drop-secondary recovery" change pattern after daily HBO treatment for 7 days in the HIBD + HBO group. The secondary recovery was not observed in the HIBD group. The post hoc test did not reveal significant differences between time-points.

\section{Discussion}

The main findings of the present study were: $\Delta \Psi \mathrm{m}$ of the ipsilateral cortex of the HIBD group within 7 days after $\mathrm{HI}$ had a 'drop-recovery-secondary drop' fluctuation, while in the HBO-treated rat pups the $\Delta \Psi \mathrm{m}$ showed a 'droprecovery-secondary drop-secondary recovery' change pattern. The secondary drop in the HIBD and HIBD + HBO groups occurred from $12 \mathrm{~h}$ to 3 days after $\mathrm{HI}$, and the extent of the secondary drop was more severe in the $\mathrm{HIBD}+\mathrm{HBO}$ group, indicating that $\mathrm{HBO}$ in the early stage after $\mathrm{HI}$ might not be a good therapy to improve mitochondrial function in the cerebral cortex. On the other hand, a secondary recovery occurred after 4 days of $\mathrm{HBO}$ treatment, elevating $\triangle \Psi \mathrm{m}$ of the ipsilateral cortex in the HIBD $+\mathrm{HBO}$ group to near normal levels. This might indicate a possible protective effect of $\mathrm{HBO}$ treatment in $\mathrm{HI}$-induced brain damage by improving neural cell mitochondrial function. These findings add to the understanding of the effects of $\mathrm{HBO}$ in neonatal HIBD, and its relevant mechanisms.

$\Delta \Psi \mathrm{m}$ is a commonly used surrogate biomarker for mitochondrial function. After brain injury, there is a cascade of biochemical events leading to mitochondrial dysfunction (15). In the present study, Rho123 was used as a probe for mitochondrial function. Rho123 is a cationic fluorescent dye that binds specifically to the mitochondria of living cells, and it has been used for the estimation of $\Delta \Psi \mathrm{m}(16)$. Another commonly used fluorescent probe for estimating $\Delta \Psi \mathrm{m}$ is $5,5^{\prime}, 6,6^{\prime}$-tetrachloro-1, $1^{\prime}, 3,3^{\prime}$-tetra-ethylbenzimidazol-carbocyanine iodide (JC-1) (17). Fluorescence

Table 2. Mitochondrial membrane potential (in mean fluorescence level, MFL) in ipsilateral cortex after a single dose of hyperbaric oxygenation (HBO) in 7-day interval after hypoxic-ischemic brain damage (HIBD).

\begin{tabular}{lcccccc}
\hline Time-point & Normal control & HIBD group & HIBD + HBO group & $t$ value & P value & $95 \% \mathrm{Cl}$ \\
\hline 1 day & $4.69 \pm 0.56$ & $3.73 \pm 0.36$ & $3.09 \pm 0.48$ & 3.25 & 0.005 & $0.22 ; 1.05$ \\
2 days & $4.78 \pm 0.42$ & $3.40 \pm 0.28$ & $2.63 \pm 1.47$ & 2.26 & 0.046 & $0.02 ; 1.52$ \\
3 days & $4.53 \pm 0.51$ & $3.35 \pm 0.91$ & $2.62 \pm 1.03$ & 2.24 & 0.038 & $0.05 ; 1.40$ \\
4 days & $4.62 \pm 0.36$ & $3.32 \pm 0.90$ & $3.50 \pm 1.34$ & -0.36 & 0.725 & $-1.26 ; 0.89$ \\
5 days & $4.67 \pm 0.42$ & $3.33 \pm 0.65$ & $3.96 \pm 0.72$ & -2.04 & 0.057 & $-1.27 ; 0.02$ \\
6 days & $4.88 \pm 0.36$ & $3.37 \pm 1.03$ & $4.55 \pm 1.82$ & -2.14 & 0.046 & $-2.35 ;-0.02$ \\
7 days & $4.79 \pm 0.28$ & $3.27 \pm 0.58$ & $4.10 \pm 2.44$ & -2.89 & 0.010 & $-1.43 ;-0.23$ \\
\hline
\end{tabular}

Data are reported as means $\pm S D$ ( $n=10$ in each group at each time-point) (ANOVA followed by Tukey's post hoc test). 


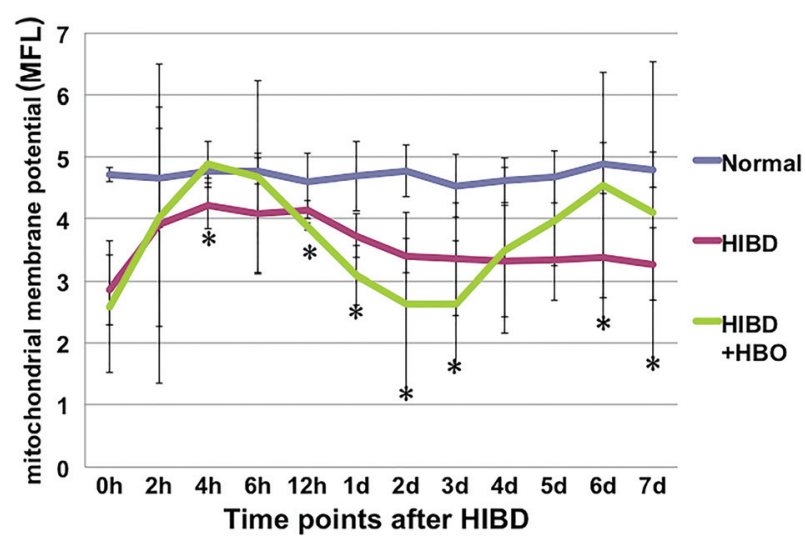

Figure 1. Change in mitochondrial membrane potential $(\Delta \psi \mathrm{m})$ in ispilateral cortex of newborn rats after hypoxic-ischemic brain damage (HIBD) and HIBD with daily hyperbaric oxygenation (HBO) treatment for 7 days. The change in $\Delta \Psi \mathrm{m}$ (mean fluorescence level, MFL) in both groups showed fluctuating pattern. Within 2 to $12 \mathrm{~h}$ after $\mathrm{HI}$ insult, $\triangle \Psi \mathrm{m}$ of the HIBD group recovered to some extent, but in the HIBD + HBO group it recovered to almost normal levels. A secondary drop (more severe in the HIBD + HBO group) was observed in both groups at 1-4 days after hypoxia-ischemia $(\mathrm{HI})$ insult. A secondary recovery was observed in the HIBD + HBO group only. Data are reported as means $\pm \mathrm{SD}$. ${ }^{*} \mathrm{P}<0.05$, significant differences between HIBD and HIBD + HBO groups (ANOVA followed by Tukey's post hoc test).

is a commonly used bioanalytical method, offering sensitive and quantitative results. The best example of the latter is flow cytometry (18), which was used in the present study. We used the Rice-Vannucci HIBD rat model, which presents the advantage of allowing the ipsilateral hemisphere of the animal to be the internal control (10).

Oxygen delivery depends on a pressure gradient from the alveolar spaces to the blood and finally to the brain tissue itself. Hyperbaric $\mathrm{O}_{2}$ increases the vital $\mathrm{O}_{2}$ delivery pressure gradient. Brain tissue $\mathrm{O}_{2}$ monitoring levels of 200-300 mm are recorded with $\mathrm{HBO}$ at 1.5 ATA (19), which increases the amount of dissolved $\mathrm{O}_{2}$ in the plasma 10-fold normal levels (20). The HBO treatment in the present study was at 2 ATA pressure for $60 \mathrm{~min}$, hence we believe that the amount of dissolved $\mathrm{O}_{2}$ in the plasma was higher than 10 -fold normal levels. This protocol is one of the most commonly used for standard therapeutic purposes (1.8-2.8 ATA for 60-90 min) (21). The increased amount of oxygen dissolved in the blood results in improvement of a variety of clinical conditions such as hypoxia (22). HBO has been used for multiple neurological diseases (23), including neonates (24). It was reported that a single dose of $\mathrm{HBO}$ reduced $\mathrm{HI}$ brain injury in neonatal rats (25). The main mechanism of HBO in HIBD might be the favorable influence in the binding of $\mathrm{O}_{2}$ in mitochondrial redox enzyme systems, which helps to improve mitochondrial function (26), and to inhibit apoptosis (27).
The findings of the present study are in accordance with other studies. Zhou et al. (9) reported that HBO improved mitochondrial function by preserving $\Delta \Psi \mathrm{m}$ and increasing ATP production after fluid-percussion brain injury. Palzur et al. (28) reported that the neuroprotective effect of HBO was mediated by inhibition of the MPTP and subsequent reduction of the mitochondrial pathway of apoptosis. This may explain the mechanism for the recovery of $\Delta \Psi \mathrm{m}$ in the ipsilateral cortex in the HIBD+ HBO group. In the present study, the more severe drop of $\Delta \Psi \mathrm{m}$ at $12 \mathrm{~h}$ to 3 days in the HIBD $+\mathrm{HBO}$ group indicates a more severe damage in mitochondrial function caused by HBO. This is not in accordance with the findings of a study in transient focal ischemia brain damage in the adult middle cerebral artery rat model, in which $\mathrm{HBO}$ was highly efficient in reducing ischemic injury within the first $6 \mathrm{~h} \mathrm{(29).}$ These results suggest that early application of $\mathrm{HBO}$ treatment might not help to reduce brain damage after $\mathrm{HI}$ in neonatal rats. We consider that this mechanism is related to tissue reperfusion and free radical damage. After brain injury, reactive oxygen and reactive nitrogen species may be generated by inflammatory cells through several different cellular pathways, including calcium activation of phospholipases, nitric oxide synthase, xanthine oxidase, and the Fenton and Haber-Weiss reactions (30). If cellular defense systems are weakened, increased production of free radicals will lead to oxidation of lipids, proteins, and nucleic acids, which may alter cellular function in a critical way (30). In addition, hyperoxia can also cause potential cerebral toxicity. Brain tissue is especially vulnerable to lipid peroxidation because of its high rate of $\mathrm{O}_{2}$ consumption and high content of phospholipids (31). Additionally, the brain has limited natural protection against free radicals - that is, it has limited reactive oxygen species (ROS) scavenging ability, poor catalase activity, and is rich in iron, which is an initiator of ROS generation in brain injury via the Fenton reaction (26).

In the neonatal population, $\mathrm{O}_{2}$ toxicity has always drawn neonatologist's attention due to its negative outcomes of retinopathy of prematurity and lung injuries. However, it was reported that HBO at 2.0 ATA for $60 \mathrm{~min}$, including intermittent decompression and compression 2-3 times per $24 \mathrm{~h}$, is a relatively low $\mathrm{O}_{2}$ exposure and carries a low risk for $\mathrm{O}_{2}$ toxicity in rats (26). In addition, exposure to hyperoxia for $1 \mathrm{~h}$ at hyperbaric pressures did not result in the structural changes or abnormal vascularization associated with retinopathy of prematurity in rats (24). Yoles et al. (32) compared the metabolic response, the hemodynamic changes and the electrical activity of dog puppies of different ages during exposure to $\mathrm{HBO}$, and found that younger puppies had higher resistance to $\mathrm{HBO}$-caused brain damage.

The limitations of the present study were: 1) sample size of the groups at each time-point might have not been large enough to reduce the SD of the data. 2) The effect of 
$\mathrm{HBO}$ to HIBD regarding memory or cognitive development was not evaluated. 3) Mechanisms of the $\Delta \Psi \mathrm{m}$ fluctuation after HBO treatment, such as NMDA receptor activation or intracellular free calcium accumulation, were not included in the present study. These mechanisms might be our next research considerations.

In conclusion, the timing and mechanisms of HBO protection in HIBD have only been partially elucidated. A fluctuation of mitochondrial function with primary and secondary drops was observed during the 1-4-day period post-HI. There was a secondary recovery of the mitochondrial function after $\mathrm{HBO}$ intervention during the 5-7-day

\section{References}

1. Glass HC, Bonifacio SL, Peloquin S, Shimotake T, Sehring $\mathrm{S}$, Sun $\mathrm{Y}$, et al. Neurocritical care for neonates. Neurocrit Care 2010; 12: 421-429, doi: 10.1007/s12028-009-9324-7.

2. Ma H, Sinha B, Pandya RS, Lin N, Popp AJ, Li J, et al. Therapeutic hypothermia as a neuroprotective strategy in neonatal hypoxic-ischemic brain injury and traumatic brain injury. Curr Mol Med 2012; 12: 1282-1296, doi: 10.2174/ 156652412803833517.

3. Pietrini D, Piastra M, Luca E, Mancino A, Conti G, Cavaliere $F$, et al. Neuroprotection and hypothermia in infants and children. Curr Drug Targets 2012; 13: 925-935, doi: 10.2174/ 138945012800675641.

4. Vert P, Daval JL. [Cell death and neurogenesis after hypoxia: a brain repair mechanism in the developing rat?]. Bull Acad Natl Med 2006; 190: 469-481.

5. Cooper CE. In vivo measurements of mitochondrial function and cell death following hypoxic/ischaemic damage to the new-born brain. Biochem Soc Symp 1999; 66: 123-140, doi: 10.1042/bss0660123.

6. Zsurka G, Kunz WS. Mitochondrial dysfunction and seizures: the neuronal energy crisis. Lancet Neurol 2015; 14: 956-966, doi: 10.1016/S1474-4422(15)00148-9.

7. Pathak D, Berthet $A$, Nakamura K. Energy failure: does it contribute to neurodegeneration? Ann Neurol 2013; 74: 506-516, doi: 10.1002/ana.24014.

8. Sukoff $M H$, Ragatz RE. Hyperbaric oxygenation for the treatment of acute cerebral edema. Neurosurgery 1982; 10 : 29-38, doi: 10.1227/00006123-198201000-00006.

9. Zhou Z, Daugherty WP, Sun D, Levasseur JE, Altememi N, Hamm RJ, et al. Protection of mitochondrial function and improvement in cognitive recovery in rats treated with hyperbaric oxygen following lateral fluid-percussion injury. J Neurosurg 2007; 106: 687-694, doi: 10.3171/jns.2007. 106.4.687.

10. Rice JE III, Vannucci RC, Brierley JB. The influence of immaturity on hypoxic-ischemic brain damage in the rat. Ann Neurol 1981; 9: 131-141, doi: 10.1002/ana.410090206.

11. Hei MY, Tao HK, Tang Q, Yu B, Zhao LL. Decreased levels of pNR1 S897 protein in the cortex of neonatal Sprague Dawley rats with hypoxic-ischemic or NMDA-induced brain damage. Braz J Med Biol Res 2012; 45: 962-967, doi: 10.1590/S0100-879X2012007500100.

12. Ming-Yan $H$, Luo $Y L$, Zhang $X C$, Liu $H$, Gao R, Wu JJ. Hypoxic-ischemic injury decreases anxiety-like behavior in rats period after $\mathrm{HI}$ insult. Findings of this study indicate that HBO therapy might not improve neural cell mitochondrial function in the cerebral cortex in the early stages post-HI, but improvement might be achieved in the sub-acute stage post-HI.

\section{Acknowledgments}

This study was supported by Hu-Nan Provincial Natural Science Foundation (\#11JJ6067) and 125-Ren-Cai Foundation of the Third Xiangya Hospital of Central South University.

when associated with loss of tyrosine-hydroxylase immunoreactive neurons of the substantia nigra. Braz J Med Biol Res 2012; 45: 13-19, doi: 10.1590/S0100-879X2011007500161.

13. Hei M, Luo $Y$, Zhang X, Liu F. Tanshinone Ila alleviates the biochemical changes associated with hypoxic ischemic brain damage in a rat model. Phytother Res 2011; 25 : 1865-1869, doi: 10.1002/ptr.3500.

14. Maric D, Maric I, Barker JL. Developmental changes in cell calcium homeostasis during neurogenesis of the embryonic rat cerebral cortex. Cereb Cortex 2000; 10: 561-573, doi: 10.1093/cercor/10.6.561.

15. Signoretti S, Marmarou A, Aygok GA, Fatouros PP, Portella $G$, Bullock RM. Assessment of mitochondrial impairment in traumatic brain injury using high-resolution proton magnetic resonance spectroscopy. J Neurosurg 2008; 108: 42-52, doi: 10.3171/JNS/2008/108/01/0042.

16. Sureda FX, Escubedo E, Gabriel C, Comas J, Camarasa J, Camins A. Mitochondrial membrane potential measurement in rat cerebellar neurons by flow cytometry. Cytometry 1997; 28: 74-80, doi: 10.1002/(SICI)1097-0320(19970501)28:1 $<74:: A I D-C Y T O 9>3.0 . C O ; 2-H$.

17. Liu J, Yu Z, Guo S, Lee SR, Xing C, Zhang C, et al. Effects of neuroglobin overexpression on mitochondrial function and oxidative stress following hypoxia/reoxygenation in cultured neurons. J Neurosci Res 2009; 87: 164-170, doi: 10.1002/ jnr.21826.

18. Nolan JP, Duggan E, Liu E, Condello D, Dave I, Stoner SA. Single cell analysis using surface enhanced Raman scattering (SERS) tags. Methods 2012; 57: 272-279, doi: 10.1016/j.ymeth.2012.03.024.

19. Daugherty WP, Levasseur JE, Sun D, Rockswold GL, Bullock MR. Effects of hyperbaric oxygen therapy on cerebral oxygenation and mitochondrial function following moderate lateral fluid-percussion injury in rats. J Neurosurg 2004; 101: 499-504, doi: 10.3171/jns.2004.101.3.0499.

20. Jain K, Baydin S. Textbook of hyperbaric medicine. 3rd edn. Seattle: Hogrefe \& Huber; 1999.

21. Benedetti S, Lamorgese A, Piersantelli M, Pagliarani S, Benvenuti F, Canestrari F. Oxidative stress and antioxidant status in patients undergoing prolonged exposure to hyperbaric oxygen. Clin Biochem 2004; 37: 312-317, doi: 10.1016/j.clinbiochem.2003.12.001.

22. Lipsky BA, Berendt AR, Deery HG, Embil JM, Joseph WS, Karchmer AW, et al. Diagnosis and treatment of diabetic foot 
infections. Clin Infect Dis 2004; 39: 885-910, doi: 10.1086/ 424846 .

23. Ostrowski RP, Colohan AR, Zhang JH. Neuroprotective effect of hyperbaric oxygen in a rat model of subarachnoid hemorrhage. Acta Neurochir Suppl 2006; 96: 188-193, doi: 10.1007/3-211-30714-1.

24. Calvert JW, Zhou C, Zhang JH. Transient exposure of rat pups to hyperoxia at normobaric and hyperbaric pressures does not cause retinopathy of prematurity. Exp Neurol 2004; 189: 150-161, doi: 10.1016/j.expneurol.2004.05.030.

25. Freiberger JJ, Suliman HB, Sheng H, McAdoo J, Piantadosi CA, Warner DS. A comparison of hyperbaric oxygen versus hypoxic cerebral preconditioning in neonatal rats. Brain Res 2006; 1075: 213-222, doi: 10.1016/j.brainres.2005.12.088.

26. Freiberger JJ, Suliman HB, Sheng H, McAdoo J, Piantadosi CA, Warner DS. A comparison of hyperbaric oxygen versus hypoxic cerebral preconditioning in neonatal rats. Brain Res 2006; 1075: 213-222, doi: 10.1016/j.brainres.2005.12.088.

27. Lou M, Chen $Y$, Ding M, Eschenfelder CC, Deuschl G. Involvement of the mitochondrial ATP-sensitive potassium channel in the neuroprotective effect of hyperbaric oxygenation after cerebral ischemia. Brain Res Bull 2006; 69: 109-116, doi: 10.1016/j.brainresbull.2005.11.009.

28. Palzur E, Zaaroor M, Vlodavsky E, Milman F, Soustiel JF. Neuroprotective effect of hyperbaric oxygen therapy in brain injury is mediated by preservation of mitochondrial membrane properties. Brain Res 2008; 1221: 126-133, doi: 10.1016/j.brainres.2008.04.078.

29. Lou M, Eschenfelder CC, Herdegen T, Brecht S, Deuschl G. Therapeutic window for use of hyperbaric oxygenation in focal transient ischemia in rats. Stroke 2004; 35: 578-583, doi: 10.1161/01.STR.0000111599.77426.A0.

30. Lewen A, Matz P, Chan PH. Free radical pathways in CNS injury. J Neurotrauma 2000; 17: 871-890, doi: 10.1089/ neu.2000.17.871

31. Demopoulos HB, Flamm ES, Seligman ML, Pietronigro DD. Oxygen free radicals in central nervous system ischemia and trauma. In: AP (Editor), Pathology of oxygen. New York: Academic Press; 1982. p 127-155.

32. Yoles $E$, Zurovsky $Y$, Zarchin N, Mayevsky A. The effect of hyperbaric hyperoxia on brain function in the newborn dog in vivo. Neurol Res 2000; 22: 404-408. 